\title{
RECORD TIMES
}

\section{J. GALAMBOS AND E. SENETA}

ABSTRACT. Let $X_{1}, X_{2}, \cdots$ be independent and identically distributed random variables with a continuous distribution function. $L(n) \geq 2$ is called a record time if $X_{L(n)}$ is strictly larger than any previous $X_{j}$, and we put $L(1)=1$. The sequence $1=L(1)<L(2)<L(3)<\cdots$ of record times is a strictly increasing sequence of random variables. In the present note we investigate the sequence $\{L(n)\}$ through the ratios $U(n)=L(n) / L(n-1), n \geq$ 2. We use an integer valued approximation $T(n)$ to $U(n)$, defined as the smallest integer such that $U(n) \leq T(n)$. These approximations turn out to be independent and identically distributed. This fact makes it possible to deduce several limit laws for $U(n)$ and for $\Delta(n)=L(n)-L(n-1), n \geq 2$.

1. The results. Let $X_{1}, X_{2}, \ldots$ be independent and identically distributed (i.i.d.) random variables with a common continuous distribution function $F(x)$. We define the record times $L(n)$ by $L(1)=1$ and

$$
L(n)=\min \left\{j: X_{j}>X_{L(n-1)}\right\}, \quad n \geq 2 .
$$

Evidently, the functions $L(n)$ are random variables and are well defined by the assumption of continuity of $F(x)$. It is easy to see that the distribution of $L(n)$ does not depend on $F(x)$. Hence, the distribution of $L(n)$ can be determined by considering the uniform distribution $F(x)=x$ for $0 \leq x \leq 1$. This immediately yields that the sequence $L(2), L(3), \ldots$ forms a homogeneous Markov chain with initial distribution

$$
P(L(2)=j)=1 / j(j-1), \quad j \geq 2,
$$

and with transition probabilities

$$
P(L(n)=k \mid L(n-1)=j)=j / k(k-1) \text { for } k \geq j+1 \geq n \geq 3,
$$

and the conditional probabilities above are equal to zero for any other values of $j$ and $k$. This elementary fact was first recognized by Rényi [3], and he

Received by the editors November 15, 1973 and, in revised form, March 18, 1974. AMS (MOS) subject classifications (1970). Primary 60F 05; Secondary 60J10, $62 \mathrm{G} 30$.

Key words and phrases. Continuous distribution, record times, ratios of record times, limit laws, maximum. 
himself deduced several of its consequences; further research has since been done by several mathematicians. Out of the papers on this topic, see the most recent ones [4], [5] and [6, p. 81], and their references, or, alternatively, the forthcoming survey [7].

In the present paper, we investigate the sequence $L(n)$ through the ratios $U(n)=L(n) / L(n-1), n \geq 2$. Though several results are known for the sequence $U(n)$, the following statements appear to be new. Our results and our investigation were guided by a recent result [2] by one of us in metric number theory. The fact that record times are related to a series expansion, called Engel's series, has been noted earlier by Rényi [3] and Vervaat [6]. Of course, we do not need this relation and we shall make no further reference to it.

We formulate our main result as a theorem.

Theorem. Let the integer valued function $T(n)$ be defined by the inequalities $T(n)-1<U(n) \leq T(n), n \geq 2$. Then the random variables $T(n)$ are i.i.d. with

$$
P(T(n)=j)=1 / j(j-1), \quad j \geq 2 .
$$

Several consequences of our theorem are worth recording. We shall deduce that, as $n \rightarrow+\infty$,

$$
[U(2)+U(3)+\cdots+U(n)] / n \log n \rightarrow 1
$$

in probability, and that, for any real $z>0$,

$$
\lim P(\max \{U(2), U(3), \cdots, U(n)\}<n z)=\exp (-1 / z) .
$$

In another direction, our theorem can be applied to compare the gaps

$$
\Delta(n)=L(n)-L(n-1), \quad n \geq 2,
$$

with $L(n-1)$. Indeed, observing that, for any positive integer $s$,

$$
\{\Delta(n)>s L(n-1)\}=\{T(n)>s+1\},
$$

several results are immediate. For example, if $a(N, s)$ denotes the number of integers $2 \leq n \leq N$ for which the inequality on the left-hand side of (5) holds, the Theorem and (5), in view of the strong law of large numbers, imply that, as $N \rightarrow+\infty$,

$$
P\left(\lim \frac{a(N, s)}{N}=\frac{1}{s+1}\right)=1 \text {. }
$$

In particular, the case $s=1$ yields that, almost surely, the number of integers 
$n \leq N$ for which $\Delta(n)>L(n-1)$ is approximately equal to those for which $\Delta(n) \leq L(n-1)$. By the central limit theorem and the iterated logarithm theorem, further information on $a(N, s)$ is immediate. On the other hand, the choice of $s$ in (5) as a function $s(n)$ of $n$ leads to previously known limit results. However, it was not our aim to reobtain known results, hence we do not give further details.

In concluding this section, we remark that the limit in (3) can not be replaced by a strong limit law, as we shall see at the end of the next section.

\section{Proofs.}

Proof of the Theorem. Notice that, by the definition of $T(n)$, the event $\{T(n)>j\}$ is equivalent to $\{U(n)>j\}$ for any integer $j \geq 1$. Thus

$$
\begin{aligned}
P\left(T(2)>j_{2},\right. & \left.T(3)>j_{3}, \cdots, T(n)>j_{n}\right) \\
= & P\left(U(2)>j_{2}, U(3)>j_{3}, \cdots, U(n)>j_{n}\right) \\
& =\sum_{n} P\left(L(2)=k_{2}, L(3)=k_{3}, \cdots, L(n)=k_{n}\right),
\end{aligned}
$$

where $\Sigma_{n}$ is summation over all $(n-1)$-vectors $\left(k_{2}, k_{3}, \ldots, k_{n}\right)$ for which $k_{2}>j_{2}$ and $k_{t}>j_{t} k_{t-1}$ for $3 \leq t \leq n$. We first observe that

$$
\begin{aligned}
& \sum_{n} P\left(L(2)=k_{2}, L(3)=k_{3}, \cdots, L(n)=k_{n}\right) \\
& \quad=\sum_{n-1} P\left(L(2)=k_{2}, L(3)=k_{3}, \cdots, L(n-1)=k_{n-1}, L(n)>j_{n} k_{n-1}\right) .
\end{aligned}
$$

On the other hand, by the L's forming a Markov chain and by (2) we have (putting $\Sigma^{*}$ for summation over $r$ with $j_{n} k_{n-1}<r<+\infty$ )

$$
\begin{aligned}
P(L(2) & \left.=k_{2}, L(3)=k_{3}, \cdots, L(n-1)=k_{n-1}, L(n)>j_{n} k_{n-1}\right) \\
& =P\left(L(2)=k_{2}, L(3)=k_{3}, \cdots, L(n-1)=k_{n-1}\right) \sum^{*} \frac{k_{n-1}}{r(r-1)} \\
& =\left(1 / j_{n}\right) P\left(L(2)=k_{2}, L(3)=k_{3}, \cdots, L(n-1)=k_{n-1}\right) .
\end{aligned}
$$

This, in view of (6) and (7), gives that

$$
\begin{aligned}
& P\left(T(2)>j_{2},\right.\left.T(3)>j_{3}, \cdots, T(n)>j_{n}\right) \\
&=\left(1 / j_{n}\right) P\left(T(2)>j_{2}, \cdots, T(n-1)>j_{n-1}\right),
\end{aligned}
$$

and thus by induction we get that

$$
P\left(T(2)>j_{2}, T(3)>j_{3}, \cdots, T(n)>j_{n}\right)=1 /\left(j_{2} j_{3} \cdots j_{n}\right) .
$$


The above equation implies that the random variables $T(s), s \geq 2$, are independent with distribution $P(T(s)>j)=1 / j, j \geq 1$. Therefore

$$
P(T(s)=j)=P(T(s)>j-1)-P(T(s)>j)=1 / j(j-1)
$$

for any integers $s \geq 2$ and $j \geq 2$. The Theorem is thus established.

In order to prove the relations in (3) and (4), we note that, by definition, for all $s \geq 2,0 \leq T(s)-U(s)<1$. Therefore,

and

$$
0 \leq \sum_{s=2}^{n} T(s)-\sum_{s=2}^{n} U(s)<n
$$

$$
0 \leq \max \{T(s): 2 \leq s \leq n\}-\max \{U(s): 2 \leq s \leq n\}<1
$$

Now, since, as $n \rightarrow+\infty$,

$$
T(2)+T(3)+\cdots+T(n) \sim n \log n
$$

in probability, which easily follows from the Theorem by turning to characteristic functions, and, also by our Theorem,

$$
P(\max \{T(s): 2 \leq s \leq n\}<n z)=P^{n-1}(T(s)<n z) \sim \exp (-1 / z),
$$

(8) and (9) immediately yield (3) and (4). (In the above argument, we could have referred to existing theory of limit laws for i.i.d. random variables, but in this simple concrete case, a direct proof is very easy.)

The fact that the limit in (3) can not be replaced by an almost sure convergence follows from the result of [1] and from (8). As a matter of fact, if (3) were true with probability one, then (8) would imply a similar limit law for the $T$ 's, which are i.i.d. with infinite expectation. This however contradicts the general result of [1].

From the inequalities (8) and (9), further limit laws can be deduced. They actually express the fact that, in order to investigate the $U$ 's, we can turn to the independent $T^{\prime}$ s. For example, putting $U^{*}(n)=\max \{U(j): 2 \leq j$ $\leq n\}$, we have that, with probability one, $\log U^{*}(n) \sim \log n$ as $n \rightarrow+\infty$.

\section{REFERENCES}

1. Y. S. Chow and H. Robbins, On sums of independent random variables with infinite moments and "fair" games, Proc. Nat. Acad. Sci. U.S.A. 47 (1961), 330-335. MR 23 \#A2908.

2. J. Galambos, Further ergodic results on the Oppenheim series, Quart. J. Math. Oxford Ser. 25 (1974), 135-141.

3. A. Rényi, Théorie des éléments saillants d'une suite d'observations, Actes du Colloq. de Math. Rényi à Clermont à l'Occasion du Tricentenaire de la Mort de Blaise Pascal, Tome II, Ann. Fac. Sci. Univ. Clermont-Ferrand No. 8 (1962), 7-13. MR 44 \#3376. 
4. S. I. Resnick, Record values and maxima, Ann. Probability 1 (1973), 650-662.

5. R. W. Shorrock, On record values and record times, J. Appl. Probability 9 (1972), 316-326.

6. W. Vervaat, Success epochs in Bernoulli trials with applications in number theory, Mathematisch Centrum Tracts 42, Amsterdam, 1972.

7. —_, Limit theorems for partial maxima and records (to appear).

DEPARTMENT OF MATHEMATICS, TEMPLE UNIVERSITY, PHILADELPHIA, PENNSYLVANIA 19121

STATISTICS DEPARTMENT, S.G.S., THE AUSTRALIAN NATIONAL UNIVERSITY, BOX 4, P. O., CANBERRA 2600, AUSTRALIA 